\section{International Scientific Journal Theoretical \& Applied Science}

p-ISSN: 2308-4944 (print) $\quad$ e-ISSN: 2409-0085 (online)

Year: 2015 Issue: 12 Volume: 32

Published: $30.12 .2015 \quad$ http://T-Science.org

SECTION 27. Transport.
Andrey Stanislavovich Reshenkin

professor candidate of technical sciences, head of department

Don State Technical University, Russia v.serge.79@mail.ru

Sergey Sergeevich Vorobyev assistant professor

candidate of technical sciences

Don State Technical University, Russia

Ruslan Nersesovich Aslanjan student

Don State Technical University, Russia

Tohir Kamilzhonovich Sattarov

student

Don State Technical University, Russia

Michail Olegovich Antipanov student

Don State Technical University, Russia

\title{
STATISTICAL MODEL OF FORECASTING POSTAL CARGO FLOWS BASED ON THE PRINCIPLES OF LOGISTICS
}

Abstract: In the article we offer the justification of the forecasting model of postal cargo traffics for the postal network, using the results of statistical data on the functioning of the post offices and the entire network. We present the algorithm of the development of such a model in order to improve the quality of postal services and to reduce the cost of postal transportation on the territory of Rostov region.

Key words: statistical model, algorithm, improvement of the quality of services, reduction of the cost of transportations.

Language: Russian

Citation: Reshenkin AS, Vorobyev SS, Aslanjan RN, Sattarov TK, Antipanov MO (2015) STATISTICAL MODEL OF FORECASTING POSTAL CARGO FLOWS BASED ON THE PRINCIPLES OF LOGISTICS. ISJ Theoretical \& Applied Science 12 (32): 28-33.

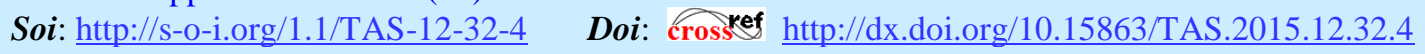

\section{СТАТИСТИЧЕСКАЯ МОДЕЛЬ ПРОГНОЗА ПОЧТОВЫХ ГРУЗОПОТОКОВ, ОСНОВАННАЯ НА ПРИНЦИПАХ ЛОГИСТИКИ}

Аннотация: В статье предложено обоснование модели прогноза грузопотоков почтовых отправлений для почтовой сети с использованием статистических данных по результатам функиионирования предприятий связи и всей сети в ичелом. Представлен алгоритм разработки такой модели с иелью повышения качества оказания почтовых услуг и снижения себестоимости почтовых перевозок по территории Ростовской области.

Ключевые слова: статистическая модель, алгоритм, повышение качества услуг, снижение себестоимости перевозок.

С развитием и становлением рыночных отношений в почтовой связи возникла острая необходимость реструктуризации почтовой подотрасли в направлении повышения качества почтовых услуг, оказываемых клиентам, с учётом максимизации возможностей предприятий связи в условиях глобализации, ужесточения конкуренции и ускорении перемен на почтовом рынке.

Учитывая, что современная почтовая связь представляет собой систему коммуникационного обслуживания юридических и физических лиц путём приёма, обработки, пересылке и доставки почтовых отправлений то, в соответствие с концепцией реструктуризации [1], достижение 
требуемого качества, можно получить за счёт повышение скорости пересылки, доставки почтовых отправлений и доступности почтовых услуг, а также, за счёт сохранения числа и технического переоснащения отделений почтовой связи филиалов федерального государственного унитарного предприятия (ФГУП) «Почта России».

При этом анализ основных положений концепции реструктуризации почтовой подотрасли свидетельствует о том, что практическая их реализация основывается на методологической основе распределительной логистики, которая рассматривает почтовую связь, как распределительную систему, состоящую из совокупности взаимодействующих разномасштабных звеньев, объединённых в единую структуру по территориальноадминистративному признаку [2].

При создании модели прогноза почтовых грузопотоков необходимо учитывать, что в почтовой связи в каждом производственном цикле выполняются следующие фазы: потребление, производство, потребление. И суть производственного процесса состоит в обеспечении передачи почтовых отправлений (ПО) от отправителя к адресату.

Практически производственный цикл включает выполнение следующих логистических функций: приём ПО, их обработку, транспортировку и доставку. Особенностью почтового производства является целостность выполнения всех перечисленных операций в едином технологическом процессе. Это наглядно представлено на рисунке 1.

Анализ структуры представленной на рисунке 1 позволяет выделить базовый элемент в рассматриваемом почтовом едином технологическом процессе - процесс транспортировки ПО, практическая реализация которого позволяет осуществить почтовую услугу по доставке ПО от отправителя к адресату в пространстве и времени.

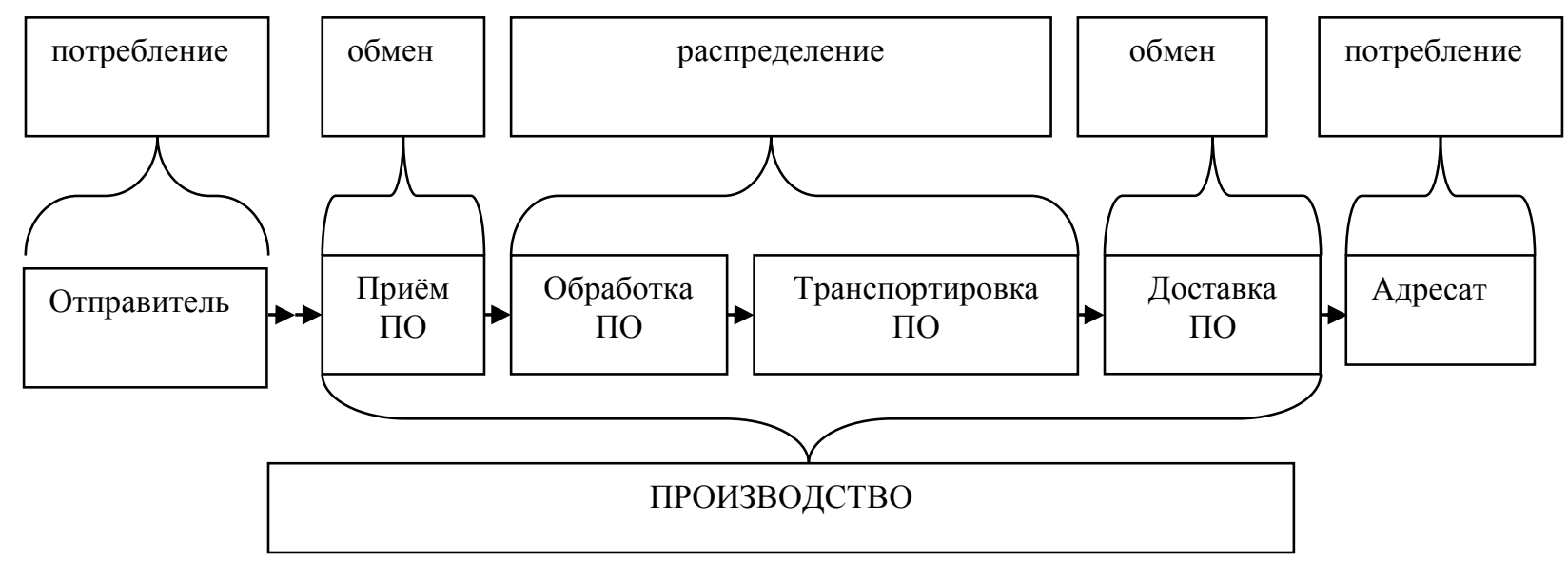

Рисунок 1 - Почтовый производственный цикл.

Анализ исследований методологической базы, проведенный отечественными и зарубежными специалистами по логистике, позволяет нам рассматривать почтовые грузопотоки ПО, как разного вида материальные потоки, которые образуются в результате транспортировки, временного складирования и выполнения других материальных операций с почтовыми отправлениями.

В почтовой связи материальные потоки или грузопотоки образуются в отделениях почтовой связи УФПС РО, которых насчитывается 1061, и далее перемещаются в направлении 50-ти районных почтамтов.

Согласно принятой почтовой классификации, грузопотоки подразделяются на внутренние, циркулирующие внутри территории
Ростовской области и внешние, уходящие за её приделы. В свою очередь внутренние грузопотоки подразделяются на исходящие и входящие.

В данном случае нас интересуют внутренние грузопотоки- исходящие и входящие, организацию которых обеспечивает УФПС РО.

Эффективность управления такими грузопотоками требует сбора, обработки и передачи информации в УФПС РО для принятия наиболее оптимального с точки зрения экономичности решения по реализации почтовой услуги. Для решения этой задачи нами предлагается использовать статистическую модель прогноза почтовых грузопотоков, построенную на принципах логистики. 
Очевидно, что в предлагаемой модели необходимо учесть роль автопредприятия (АТП), занимающихся оказанием транспортной услуги УФПС РО по доставке ПО адресатам. При этом с одной стороны АТП является элементом логистической цепи, который обеспечивает связь между звеньями макрологистической системы УФПС РО (продвижение материальных потоков в виде ПО), а с другой АТ - потребитель отдельных материальных потоков (горючее, запасные части и т.д.), конечное звено логистической цепи.

В связи с этим, представляется, что эффективность перемещения грузопотоков ПО во многом зависит от решения таких задач, как: выбор вида и типа транспортных средств; определение рациональных маршрутов доставки ПО.

Как отмечают специалисты в этом, и есть логистический подход решения задачи по скорейшей доставки адресату ПО с минимальными совокупными издержками.

При создании статистической модели прогноза почтовых грузопотоков необходимо учесть следующие условия [3]:

- системный подход к решению рассматриваемой проблемы;

- адекватность модели реальной системе, объективный учёт взаимосвязанных подсистем;

- гибкая многовариантность, т. е. согласование материальных, финансовых и информационных потоков;

- непрерывности процесса внедрения модели;

-формирование и оптимизация модели реальной системе во взаимосвязи.

Анализ литературы, посвящённой организации перемещения грузопотоков, позволяет сказать, что при разработке моделей нами использовалась идея о том, что данная модель должна позволять анализировать две взаимосвязанные характеристики процесса: состояние и функционирование.

Состояние грузопотоков характеризуется их объёмами и структурой. А функционирование перемещением грузопотоков ПО в условиях внешней среды в направлении потребителя (адресата).

При этом предполагается, что к задачам функционирования относятся: выбор оптимальных вариантов организации перевозочных процессов, видов и типов транспортных, производственных и складских процессов.

Практика свидетельствует, что оптимизация одновременно функционирование и состояние грузопотока - главное условие эффективной работы модели.
Причём предлагаемая модель должна позволять принимать управленческое решение с учётом состояния автомобильного парка, иначе нарушается основной принцип логистики надёжность.

Следует отметить, что решить задачу развития УФПС РО с помощью одной модели невозможно, поэтому для каждой подзадачи необходимо создавать свою модель, которая должна постоянно с учётом изменений внешней среды корректироваться.

Однако с учётом того, что стоимость транспортных расходов в процессе производства и реализации продукции доходит до $1 / 3$ цены конечного продукта [4], то актуальность решения вопросов по рационализации, а лучше оптимизации транспортных, погрузочноразгрузочных, тароупаковочных, экспедиционных и складских операций остается неизменной и по- прежнему, сильно влияет на снижение издержек и экономию ресурсов.

Как показывает опыт создания аналогичных моделей, основными показателями разрабатываемой модели должны быть:

- уровень эксплутационных расходов (себестоимость перевозок);

- капитальные вложения;

- скорость движения и сроки доставки;

- регулярность перевозок;

- гарантия сохранности грузов,

Актуальность разработки модели прогноза грузопотоков ПО обусловлено тем, что источником издержек в любой логистической системе является циркулирующий материальный поток (грузопоток), причём основная доля в этих издержках $(46 \%)$ составляют транспортные расходы [5].

Разрабатываемая статистическая модель прогноза почтовых грузопотоков должна позволять решать множество задач, связанных с организацией перемещения почтовых грузов. Такие как выбор числа транспортных средств, их типа и размеров, выборы маршрутов, распределение грузов по транспортным средствам, графики обслуживания транспортных средств.

Основная проблема маршрутизации связана с выбором во времени поездки лучшего пути для доставки почтовой продукции адресатам.

Существует множество вариантов этой проблемы, которые в любом случае оптимально решать трудно. Существует базовая задача выбора маршрута - «задача коммивояжёра». Решения реальной задачи связаны с такими факторами, как парк различных типов автомобильных средств, различные логистические характеристики, временные складирования, меняющая скорость из-за условий 
доставки, меняющееся время доставки, неопределённость с затратами.
Структурно показатель расхода на 1 тонну груза можно представить так, (рисунок 2).

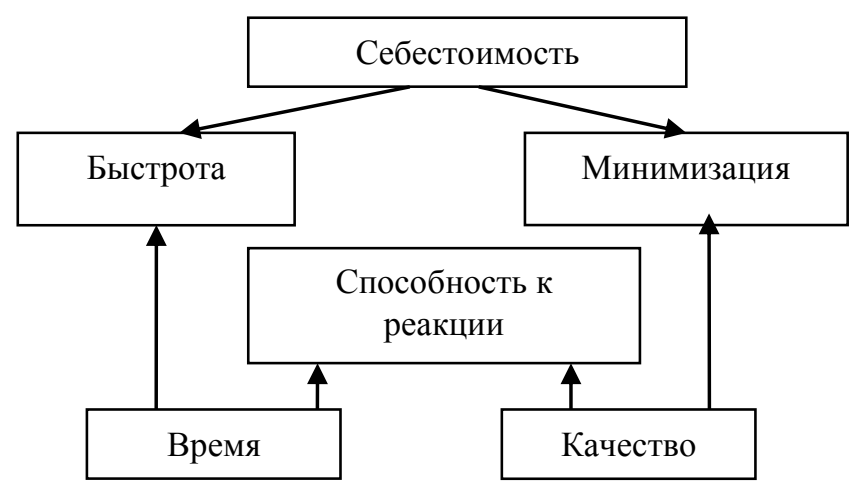

Рисунок 2 - Показатель расхода на 1 тонну груза.

Существует множество методов выбора маршрутов, но в целом выделяется два [6] .

В первом для выбора лучшего пути используется географические программы, не учитывающие реально имеющиеся дороги.

Второй подход анализирует дорожную сеть и позволяет отыскивать кратчайший путь между заданными точками.

Существуют следующие методы маршрутизации:

-корректировка предыдущих планов, связанных с привлечением специалистов;

-интуитивные методы;

-использование картограмм;

-математические модели, позволяющие получать оптимальные решения без вмешательства человека.

Нами предлагается создание математической модели в виде статистическая модели прогноза почтовых грузопотоков, которая позволяет оценить оптимальность маршрутов по вариациям затрат при незначительных изменениях маршрутов.

В этом случае учитывая, что расстояние выбранного маршрут постоянная величина (const), а переменные величины: грузопоток (P) переменная (var 1); времена года (B)- переменная (var 2); состояние дорог (Д) - переменная (var 3), получаем зависимость:

$$
M=F(\mathrm{P}, \mathrm{B}, \text { Д })
$$

В настоящее время повышение конкуренции на почтовом рынке требует повышение качества выполнения почтовой услуги по доставке ПО адресатам, что связано с сокращением времени их транспортировки. Причём, если сокращение времени транспортировки ПО является необходимым условием повышения эффективности работы предприятий связи УФПС PO, то минимизация совокупных издержек, связанных с перемещением ПО, на пути от отправителя до адресата - достаточным. В свою очередь оптимизация рассматриваемых совокупных издержек связано с оптимизацией такой величины, как совокупная величина себестоимости транспортировки почтовых отправлений по территории РО. Предлагаемый логистический подход при создании статистическая модель прогноза почтовых грузопотоков позволяет осуществит сквозную оптимизацию суммарных себестоимостей при осуществлении транспортировки почтовых грузов на всём пути их движения. Причём для разработки этой модели нами предлагается использовать методологию функциональностоимостного анализа (ФСА) [7].

$\mathrm{B}$ соответствии с общей теорией логистики оптимальным считается такой уровень себестоимости перевозок, при котором полностью реализуется заданные параметры грузопотоков ПО с минимальными затратами.

Таким образом, использование ФСА, в конечном счете, позволяет не столько снизить себестоимость транспортировки ПО, сколько осуществить организационно-технологическую оптимизацию логистических процессов в целом в цепи поставки ПО.

Использование ФСА при разработке статистической модели прогноза почтовых грузопотоков даёт возможность анализировать не только сам технологический процесс почтового производства, но и осуществлять оптимизацию логистических издержек, в частности себестоимости перевозок [8].

При этом, использование ФСА в данном случае предполагает пошаговое идентифицирование, анализ и оценка функционально-стоимостного содержания логистической деятельности в почтовом 


\begin{tabular}{|c|c|c|c|c|c|c|}
\hline Impact Factor: & $\begin{array}{l}\text { ISRA (India) } \\
\text { ISI (Dubai, UAF } \\
\text { GIF (Australia) } \\
\text { JIF }\end{array}$ & $\begin{array}{l}=1.344 \\
=0.829 \\
=0.564 \\
=1.500\end{array}$ & $\begin{array}{l}\text { SIS (USA) } \\
\text { PИНЦ (Russia) } \\
\text { ESJI (KZ) } \\
\text { SJIF (Morocco) }\end{array}$ & $\begin{array}{l}=0.912 \\
=0.179 \\
=1.042 \\
=2.031\end{array}$ & $\begin{array}{l}\text { ICV (Poland) } \\
\text { PIF (India) }\end{array}$ & $\begin{array}{l}=6.630 \\
=1.940\end{array}$ \\
\hline
\end{tabular}

производстве, включая этапы: подготовительный, информационный, аналитической и внедрения. Если на подготовительном этапе происходит детализация целей и задач ФСА, то на информационном производиться сбор и обобщение данных о факторах, влияющих на себестоимость перевозок в почтовой логистической цепи. После этого на аналитическом этапе с учётом структуры грузопотока ПО разрабатывается оптимальная модель их прогноза.

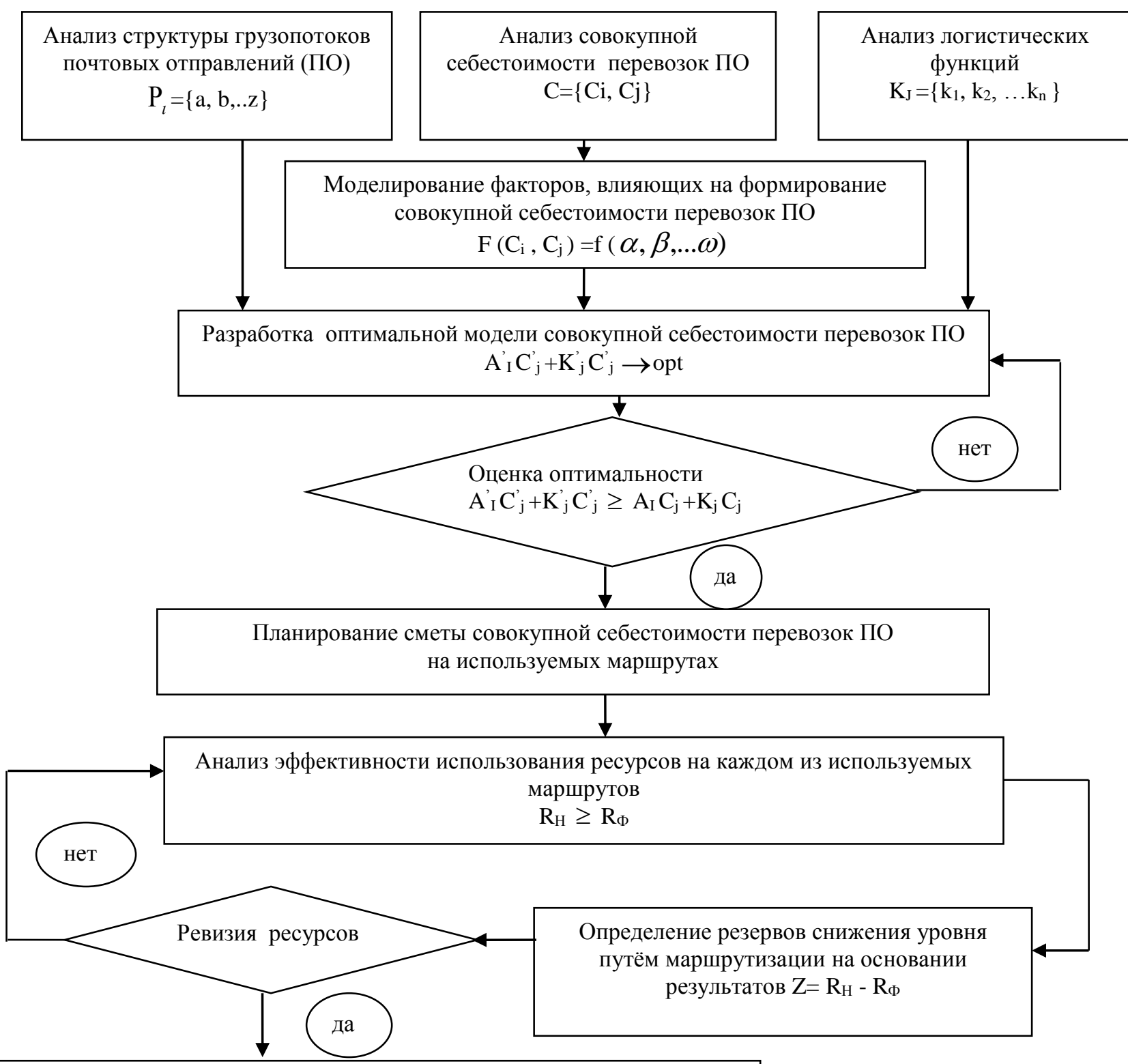

Планирование снижения уровня совокупной себестоимости перевозок ПО, путём осуществления рационализации маршрутов

Рисунок 3 - Блок - схема алгоритма проведения аналитического этапа ФСА.

Специалистами предлагается при создании модели, первоначально разрабатывать блок схему аналитического этапа ФСА [7], которая в нашем случае имеет вид, представленный на рисунке 3. При этом рассматриваем совокупную себестоимость транспортировки ПО в качестве целевой функции оптимального уровня логистических издержек. 


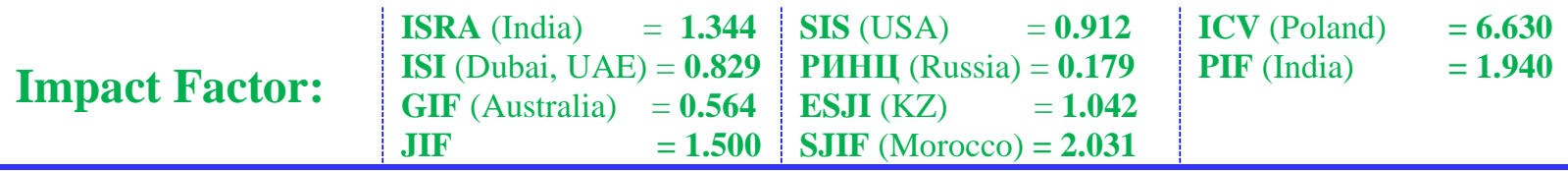

В соответствии с [6] себестоимость транспортных перевозок определяется по формуле: $\frac{C_{i}^{\text {cos. }}}{P_{i}^{\Sigma}} \rightarrow \min , \quad$ где $C^{\text {сов }}{ }_{i}-$ совокупная себестоимость транспортировки почтовых отправлений;

$$
\mathrm{P}_{i}^{\Sigma} \quad \text { - объём грузопотока почтовых }
$$

отправлений.

Обозначения: $P_{i^{-}}$индекс параметров $i$-го грузопотока почтовых отправлений; $a, b, z-$ параметры $i$-го грузопотока; $C$ - индекс совокупной себестоимости перевозок; $\mathrm{Ci}$ величина себестоимости транспортировки по $i$ ому материальному потоку; $\mathrm{Cj}-$ величина себестоимости транспортировки по $j$-ой логистической функции; $K_{j}$-качество $j$-ой функции; $\kappa_{l}, \kappa_{2} \ldots \kappa_{n}$-показатели качества логистических функций; $F_{j}\left(C_{i,} C_{J}\right)$-изменение себестоимостей транспортировки под влиянием $j$ х факторов; $f$ - функциональная зависимость и плотность связи себестоимости и факторов; ( $\alpha, \beta, \ldots \omega)$-факторы, влияющие на себестоимости транспортировки; $A^{l}{ }_{i} C^{l}{ }_{j}-$ индекс оптимальной себестоимости транспортировки по $i$-му материальному потоку; $K_{j}^{\prime} C_{j}^{\prime}-$ индекс оптимальной себестоимости транспортировки по $j$-й логистической функции; $R_{H}, R_{\Phi}-$ показатели использования ресурсов в логистических почтовых цепях, соответственно фактической и нормативной; $Z$ - резервы использования ресурсов в логистических почтовых цепях.
Из рисунка 3 видно, что осуществление ФСА означает последовательный поэтапный процесс, заключающийся в идентификации, анализе и оценки производственно-стоимостного содержания логистической деятельности в почтовом производстве всей логистической системы УФПС РО.

Сначала анализируется структура грузопотоков почтовых отправлений, затем исследуются факторы, влияющие на формирование себестоимости перевозок ПО в логистической цепи почтового производства.

Таким образом, анализ основных факторов, влияющие на формирование себестоимости перевозок ПО, позволяет формировать оптимальный бюджет по логистической функции и материальным почтовым потокам.

Кроме того, при составлении графиков транспортировки почтовых отправлений автомобильным транспортом необходимо учитывать факторы, связанные со спецификой автодорожного движения [9].

Первоначальным этапом в процессе создания модели логистической системы УФПС РО является проведение факторного анализа результатов функционирования УФПС РО, характерных причин снижения рентабельности его структурных подразделений, а так же наличия и состояния дорожно-транспортной сети Ростовской области. Кроме того, необходимо рассмотреть вопросы оптимизации и рационализации перевозочных процессов почтовой продукции по территории Ростовской области, составляющей более 100 тыс. км² [10].

\section{References:}

1. Zenkin IV (2004) Pochtovoe pravo. Akty obshhego haraktera. Moscow; central'nyj izdatel'skij DOM, 2004, pp.44.

2. Reshenkin AS, Vorobyev SS, Andros DS, Babkin VG, Ganchurin AY (2015) Logistization of the enterprises of the mail servise on the basis of the model of differentiation of service zones. ISJ Theoretical \& Applied Science 11 (31): 161-170. Doi: http://dx.doi.org/10.15863/TAS.2015.11.31.26

3. Lukinskij VS (2000) Logistika avtomobil'nogo transporta: koncepcii, metody, modeli. Moscow:Finansy i statistika, 2000, pp.44.

4. (2003) Transportnaja logistika (Pod red. Mirotina L.B. -Moscow; izd. Jekzamen, 2003, pp.51.

5. Nerush R (2003) Logistika. Moscow; JuNITDANA, 2003, pp.15.
6. Uoters D (2003) Logistika. Upravlenie cep'ju postavok: Per. s ang.- Moscow; JuNITI-DAN, 2003, pp.470.

7. Litvina DB, Tambovcev SN, Shumejko MN (2000) Logisticheskie izderzhki. Rostov-naDonu, RGSU, 2000, pp.136.

8. Karablin OV (2010) Jekonomika i tehnologija proizvodstva sistem i oborudovanija kompleksov/O.V. Karablin,A.S. Reshenkin. Rostov n/D, 2010.

9. (2014) Vvedenie v professional'nuju dejatel'nost'. Vorob'ev S.S., Vorob'ev S.A. (servis transportnyh sredstv) uchebnoe posobie / Pechataetsja po resheniju redakcionnoizdatel'skogo soveta Donskogo gosudarstvennogo tehnicheskogo universiteta. Rostov-na-Donu, 2014.

10. (2014) Rostovskaja oblast' v cifrah 2013: Stat.sb./Rostovstat.- Rostov-na-Donu, 2014. $906 \mathrm{p}$. 